\title{
Duality based boundary treatment for the Euler and Navier-Stokes equations
}

\author{
Jens Berg and Jan Nordström
}

\section{Linköping University Post Print}

\section{Tweet}

N.B.: When citing this work, cite the original article.

Original Publication:

Jens Berg and Jan Nordström, Duality based boundary treatment for the Euler and NavierStokes equations, 2013, AIAA Aerospace Sciences - Fluid Sciences Event, 1-19.

http://dx.doi.org/10.2514/6.2013-2959

From the 21st AIAA Computational Fluid Dynamics Conference, San Diego, CA, USA, 2013

Postprint available at: Linköping University Electronic Press

http://urn.kb.se/resolve?urn=urn:nbn:se:liu:diva-96812 


\title{
Duality based boundary treatment for the Euler and Navier-Stokes equations
}

\author{
Jens Berg* \\ Uppsala University, Uppsala, SE-751 05, Sweden \\ Jan Nordström ${ }^{\dagger}$ \\ Linköping University, Linköping, SE-581 83, Sweden
}

\begin{abstract}
In this paper we construct well-posed boundary conditions for the compressible Euler and Navier-Stokes equations in two space dimensions. When also considering the dual equations, we show how to construct the boundary conditions so that both the primal and dual problems are well-posed. By considering the primal and dual problems simultaneously, we construct energy stable and dual consistent finite difference schemes on summation-byparts form with weak imposition of the boundary conditions.

According to linear theory, the stable and dual consistent discretization can be used to compute linear integral functionals from the solution at a superconvergent rate. Here we evaluate numerically the superconvergence property for the non-linear Euler and NavierStokes equations with linear and non-linear integral functionals.
\end{abstract}

\section{Introduction}

Functionals can represent the lift or drag on an aircraft, energy or any other scalar quantity computed from the solution to a partial differential equation (PDE). In many engineering applications, high order accurate functionals are often of greater interest than accurate solutions of the equations themselves. Whenever there is a functional involved, the concept of duality becomes important. The solution of a PDE resides in some function space, and the set of all bounded linear functionals on that space is called its dual space. Knowledge of the functional of interest can thus be obtained by studying the dual space. This is the main topic in functional analysis and references can be found in any standard textbook.

In numerical analysis, and in particular for computational fluid dynamics problems, duality have been exploited for optimal control problems, ${ }^{1,2}$ error estimation ${ }^{3-5}$ and convergence acceleration. ${ }^{6-9}$ An extensive summary of the use of adjoint problems can be found in, ${ }^{10}$ and more recently in ${ }^{11}$ with focus on error estimation and adaptive mesh refinement.

In,${ }^{9}$ the theory of functional superconvergence was established for time-dependent problems using a finite difference method on summation-by-parts (SBP) form with boundary conditions imposed weakly by the simultaneous approximation term (SAT). In order to avoid additional theoretical difficulties, Dirichlet boundary conditions for both the primal and dual problem were used. The Dirichlet boundary conditions ensured that both problems were well-posed without additional efforts. In an Euler or Navier-Stokes calculation, however, Dirichlet boundary conditions are rarely used as far-field boundaries. Unless exact boundary data is known, Dirichlet boundary conditions cause reflections which pollute the solution. Other kind of boundary conditions are well-known to increase both the accuracy and stability properties of the scheme. ${ }^{12-14}$

When constructing boundary conditions for the primal problem, it can be beneficial to simultaneously consider the dual problem. Usually, there are a number of undetermined parameters which can be chosen more or less arbitrarily in an ad hoc way. The number and form of the boundary conditions for the dual problem usually differ from those of the primal problem. Tuning the coefficients so that both the primal and dual problems are well-posed can thus reduce the parameter space.

*Ph.D student, Department of Information Technology.

${ }^{\dagger}$ Professor, Department of Mathematics. 
In this paper, we will consider time-dependent partial differential equations of the form

$$
\begin{aligned}
u_{t}+L(u) & =f, \\
J(u) & =(g, u)
\end{aligned}
$$

where $J(u)$ is a functional output of interest, $L$ can be either linear or non-linear and $u$ can represent either a scalar or vector valued function. To find the dual problem, we follow the notation in, $, 8,9,15$ and seek a function $\theta$ in some appropriate function space, so that

$$
\int_{0}^{T} J(u) d t=\int_{0}^{T}(\theta, f) d t .
$$

A formal computation (assume that $L$ is linear and that $u$ have compact support in space) gives

$$
\begin{aligned}
\int_{0}^{T} J(u) d t & =\int_{0}^{T} J(u) d t-\int_{0}^{T}\left(\theta, u_{t}+L u-f\right) d t \\
& =\int_{0}^{T}\left(\theta_{t}-L^{*} \theta+g, u\right) d t+\int_{\Omega}[\theta u]_{0}^{T} d \Omega+\int_{0}^{T}(\theta, f) d t
\end{aligned}
$$

where $L^{*}$ is the formal adjoint, or dual, operator associated with $L$ under the inner product so that $(\theta, L u)=$ $\left(L^{*} \theta, u\right)$. By having homogeneous initial conditions for the primal problem, we obtain the time-dependent dual problem as

$$
-\theta_{t}+L^{*} \theta=g
$$

where we have to put an initial condition for the dual problem at time $t=T$. Usually one introduces the time transformation $\tau=T-t$ which transforms (4) to

$$
\theta_{\tau}+L^{*} \theta=g
$$

with an initial condition at $\tau=0$. A discretization which simultaneously approximates the primal and dual problems consistently, is called dual consistent and produces superconvergent time-dependent linear integral functionals if the scheme for the primal problem is stable. ${ }^{9}$

A discretization of the primal problem (1) can be written as

$$
\frac{d}{d t} u_{h}+L_{h} u_{h}=f
$$

where $u_{h}$ is the discrete approximation of $u$ and $L_{h}$ is a discrete approximation of $L$, including the boundary conditions. It is thus required that (6) is both stable and that the discrete dual operator, $L_{h}^{*}$, is a consistent approximation of $L^{*}$, including the dual boundary conditions.

A difference operator for the first derivative is said to be on SBP form if it can be written as

$$
D_{1}=P^{-1} Q
$$

where $P=P^{T}$ defines a norm by $\|u\|^{2}=u^{T} P u$ and $Q$ satisfies the SBP property

$$
Q+Q^{T}=E_{N}-E_{0}
$$

where

$$
E_{N}=\operatorname{diag}[0, \ldots, 0,1], \quad E_{0}=\operatorname{diag}[1,0, \ldots, 0] .
$$

The second derivative operator can be constructed either by applying the first derivative twice, i.e.

$$
D_{2}=\left(P^{-1} Q\right)^{2}
$$

which results in a wide operator, or a compact operator with minimal bandwidth of the form

$$
D_{2}=P^{-1}\left(-H+\left(E_{N}-E_{0}\right) S\right)
$$


as described in. ${ }^{16-18}$ In this paper, we consider only diagonal norms. ${ }^{19}$ A first derivative SBP operator is in essence a $2 s$-order accurate central finite difference operator which have been modified close to the boundaries so that it becomes one-sided. Together with the diagonal norm, the boundary closure is accurate of order $s$ making the SBP operator accurate of order $s+1$ in general. ${ }^{19}$ For problems with second derivatives, the compact operator can be modified with higher order accurate boundary closures to gain one extra order of accuracy. ${ }^{17,20}$

The discrete inner product in an SBP setting is defined by

$$
\left(u_{h}, v_{h}\right)_{h}=u_{h}^{T} P v_{h}
$$

and hence the discrete adjoint operator can be computed, according to the definition

$$
\left(L_{h} u_{h}, v_{h}\right)_{h}=\left(u_{h}, L_{h}^{*} v_{h}\right)_{h},
$$

as

$$
L_{h}^{*}=P^{-1} L_{h}^{T} P .
$$

The proof that a stable and dual consistent SBP scheme produces superconvergent linear functionals is presented in. ${ }^{9}$ The proof is based on the fact that the norm matrix, $P$, is a high order accurate integration operator. It was shown in ${ }^{21}$ that the matrix $P$ extends the Gregory formulas for integration and is accurate of order $2 s$. Moreover, it was shown that when integrating the numerical solution from an SBP discretization using the mass matrix $P$, you regain the full accuracy of $2 s$ for the integral.

The procedure for constructing stable schemes which superconvergent linear functionals can now be summarized as follows;

1. Determine boundary conditions so that both the primal and dual problems are well-posed

2. Discretize the primal problem and ensure stability

3. Compute $L_{h}^{*}$ and chose the remaining parameters (if any) so that the continuous adjoint $L^{*}$ is consistently approximated with the dual boundary conditions

While the procedure seems somewhat abstract, we will show using representative equations that the computations are straight forward. Note that a stable and consistent discretization of the primal problem does not imply that the dual problem is consistently approximated. In fact, that is rarely the case. Note also that dual consistency, and hence superconvergence, is merely a choice of coefficients. Superconvergence is thus obtained at no extra computational cost.

\section{The 2-D Euler equations}

We consider the non-dimensional time-dependent compressible Euler equations in two space dimensions. The non-dimensionalization have been done as

$$
\rho=\frac{\rho^{*}}{\rho_{\infty}^{*}}, \quad u=\frac{u^{*}}{c_{\infty}^{*}}, \quad v=\frac{v^{*}}{c_{\infty}^{*}}, \quad e=\frac{e^{*}}{\rho_{\infty}^{*}\left(c_{\infty}^{*}\right)^{2}}, \quad p=\frac{p^{*}}{\rho_{\infty}^{*}\left(c_{\infty}^{*}\right)^{2}}, \quad T=\frac{T^{*}}{T_{\infty}^{*}},
$$

where we have the density, velocities, energy, pressure and temperature, respectively. The $*$-superscript denotes a dimensional variable and the $\infty$-subscript the free-stream reference value. Note that the velocities are non-dimensionalized using the free-stream speed of sound, $c_{\infty}^{*}$. The equation of state is the ideal gas law in non-dimensional form,

$$
\gamma p=\rho T .
$$

More details about the non-dimensionalization can be found in i.e. ${ }^{22}$

In conservative form, the Euler equations can be written as

$$
q_{t}+F_{x}^{I}+G_{y}^{I}=0
$$

where the conservative variables, $q=[\rho, \rho u, \rho v, e]^{T}$, are the density, momentum and energy, respectively. The energy is defined by

$$
e=\frac{p}{\gamma-1}+\frac{1}{2} \rho\left(u^{2}+v^{2}\right)
$$


where $p$ is the pressure and $\gamma$ the ratio of specific heats. The fluxes are given by

$$
F^{I}=\left[\begin{array}{c}
\rho u \\
p+\rho u^{2} \\
\rho u v \\
(p+e) u
\end{array}\right], \quad G^{I}=\left[\begin{array}{c}
\rho v \\
\rho u v \\
p+\rho v^{2} \\
(p+e) v
\end{array}\right]
$$

Since (17) is a nonlinear system of equations, it is not easily analyzed using standard theory. The analysis will hence be performed on the linear, constant coefficient symmetric Euler equations in non-conservative form. This is known as the principle of linearization and localization. ${ }^{23}$ After linearizing (17), freezing the coefficients and symmetrizing, ${ }^{24}$ we obtain

$$
U_{t}+A U_{x}+B U_{y}=0
$$

where $U=\left[\frac{\bar{c}}{\sqrt{\gamma} \bar{\rho}} \rho, u, v, \frac{1}{\bar{c} \sqrt{\gamma(\gamma-1}} T\right]^{T}$ are the symmetrized variables. We abuse the notation a bit and let $\rho, u, v, T$ have the same meaning whether they are the original or linearized variables. A bar denotes a constant state around which we have linearized. The symmetric coefficient matrices, $A, B$ are found in. ${ }^{24,25}$ To simplify the analysis we let the domain of interest be the unit square, $\Omega=[0,1]^{2}$.

To determine the boundary conditions, we apply the energy method to (20). By using the Gauss-Green formula for higher-dimensional integration by parts, we obtain

$$
\begin{aligned}
\|U\|_{t}^{2} & =-\oint_{\partial \Omega} U^{T}(A U, B U) \cdot n d s \\
& =\underbrace{\int_{0}^{1} U^{T} B U d x}_{\text {south }}-\underbrace{\int_{0}^{1} U^{T} B U d x}_{\text {north }}-\underbrace{\int_{0}^{1} U^{T} A U d y}_{\text {east }}+\underbrace{\int_{0}^{1} U^{T} A U d y}_{\text {west }},
\end{aligned}
$$

and the boundary conditions for (20) have to be chosen so that

$$
\|U\|_{t}^{2} \leq 0
$$

Since the matrices $A, B$ are symmetric, we can diagonalize them as

$$
A=X \Lambda_{A} X^{T}, \quad B=Y \Lambda_{B} Y^{T}
$$

where the eigenvector matrices $X, Y$ have been normalized. By splitting $\Lambda_{A, B}$ into parts containing the positive and negative eigenvalues, respectively,

$$
\Lambda_{A}=\Lambda_{A}^{+}+\Lambda_{A}^{-}, \quad \Lambda_{B}=\Lambda_{B}^{+}+\Lambda_{B}^{-},
$$

we can estimate $(21)$ as

$$
\begin{aligned}
\|U\|_{t}^{2} & \leq \int_{0}^{1}\left(Y^{T} U\right) \Lambda_{B}^{+}\left(Y^{T} U\right) d x-\int_{0}^{1}\left(Y^{T} U\right) \Lambda_{B}^{-}\left(Y^{T} U\right) d x \\
& -\int_{0}^{1}\left(X^{T} U\right) \Lambda_{A}^{-}\left(X^{T} U\right) d y+\int_{0}^{1}\left(X^{T} U\right) \Lambda_{A}^{+}\left(X^{T} U\right) d y .
\end{aligned}
$$

From (25) we can see that we get an energy estimate if we let

$$
\Lambda_{A}^{+} X^{T} U=0, \quad \Lambda_{A}^{-} X^{T} U=0, \quad \Lambda_{B}^{+} Y^{T} U=0, \quad \Lambda_{B}^{-} X^{T} U=0 .
$$

If we define

$$
A^{+}=X^{T} \Lambda_{A}^{+} X, \quad A^{-}=X^{T} \Lambda_{A}^{-} X, \quad B^{+}=Y^{T} \Lambda_{B}^{+} Y, \quad B^{-}=Y^{T} \Lambda_{B}^{-} Y,
$$


we can write the boundary conditions in (26) as

$$
A^{+} U=0, \quad A^{-} U=0, \quad B^{+} U=0, \quad B^{-} U=0,
$$

for the west, east, south and north boundaries, respectively. The boundary conditions (28) are the characteristic boundary conditions for the Euler equations which are extensively used in CFD applications. Note that we have used homogeneous boundary conditions in the analysis. If the boundary data is included, an energy estimate can still be obtained and the problem is called strongly well-posed. ${ }^{26}$

To determine the dual operator and dual boundary conditions we let

$$
L=A \frac{\partial}{\partial x}+B \frac{\partial}{\partial y}
$$

and write $(20)$ as

$$
U_{t}+L U=0
$$

together with a linear functional of interest

$$
J(U)=(G, U)
$$

We follow the procedure $\operatorname{in}^{9,15}$ and let $U_{t}=0$, add a forcing function $F$ to (30), and seek $\theta$ so that

$$
J(U)=(\theta, F) .
$$

Integration by parts gives

$$
\begin{aligned}
J(U) & =J(U)-\int_{\Omega} \theta^{T}(L U-F) d \Omega \\
& =-\int_{\Omega} U^{T}\left(L^{*} \theta-G\right) d \Omega-\oint_{\partial \Omega} \theta^{T}(A U, B U) \cdot n d s+(\theta, F),
\end{aligned}
$$

where

$$
L^{*} \theta=-A \theta_{x}-B \theta_{y}
$$

and hence the dual operator is given by

$$
L^{*}=-A \frac{\partial}{\partial x}-B \frac{\partial}{\partial y} .
$$

We obtain the dual boundary conditions by expanding the boundary integral and applying the homogeneous boundary conditions (28) for the primal equation. The minimal number of conditions which remove the rest of the boundary terms are the dual boundary conditions. A straightforward computation results in

$$
A^{-} \theta=0, \quad A^{+} \theta=0, \quad B^{-} \theta=0, \quad B^{+} \theta=0
$$

for the west, east, south and north boundaries, respectively. Note that the dual boundary conditions (36) are the exact opposites of the primal boundary conditions (28).

\section{A. Discretization, stability and, dual consistency}

The discrete analysis will also be performed on the linear, constant coefficient and symmetric system (20). In the implementation, however, the nonlinear conservative form is used and the scheme has been transformed back to the conservative variables. For the purpose of analysis, we discretize (20) with the boundary conditions given in $(28)$ as

$$
\begin{aligned}
\frac{d}{d t} U_{h} & +\left(P_{x}^{-1} Q_{x} \otimes I_{y} \otimes A\right) U_{h}+\left(I_{x} \otimes P_{y}^{-1} Q_{y} \otimes B\right) U_{h}= \\
& +\left(P_{x}^{-1} E_{W} \otimes I_{y} \otimes \Sigma_{W}\right)\left(\left(I_{x} \otimes I_{y} \otimes A^{+}\right) U_{h}\right) \\
& +\left(P_{x}^{-1} E_{E} \otimes I_{y} \otimes \Sigma_{E}\right)\left(\left(I_{x} \otimes I_{y} \otimes A^{-}\right) U_{h}\right) \\
& +\left(I_{x} \otimes P_{y}^{-1} E_{S} \otimes \Sigma_{S}\right)\left(\left(I_{x} \otimes I_{y} \otimes B^{+}\right) U_{h}\right) \\
& +\left(I_{x} \otimes P_{y}^{-1} E_{N} \otimes \Sigma_{N}\right)\left(\left(I_{x} \otimes I_{y} \otimes B^{-}\right) U_{h}\right) .
\end{aligned}
$$


The subscripts $x, y$ indicates in which coordinate direction the operator is acting, and the subscripts $E, W, S, N$ indicates that the term acts only on the west, east, south or north boundary, respectively. The matrices $\Sigma_{W, E, S, N}$, so that the scheme is stable, is given by

Proposition II.1. The scheme (37) is stable using

$$
\Sigma_{W}=\sigma_{W} I_{4}, \quad \Sigma_{E}=\sigma_{E} I_{4}, \quad \Sigma_{S}=\sigma_{S} I_{4}, \quad \Sigma_{N}=\sigma_{N} I_{4},
$$

with

$$
\sigma_{W} \leq-\frac{1}{2}, \quad \sigma_{E} \geq \frac{1}{2}, \quad \sigma_{S} \leq-\frac{1}{2}, \quad \sigma_{N} \geq \frac{1}{2} .
$$

Proof. We apply the energy method by multiplying (37) with $U^{T}\left(P_{x} \otimes P_{y} \otimes I_{4}\right)$. We get

$$
\begin{aligned}
\left\|U_{h}\right\|_{t}^{2} & =U_{h}^{T}\left(E_{W} \otimes P_{y} \otimes\left(A+2 \Sigma_{W} A^{+}\right)\right) U_{h} \\
& +U_{h}^{T}\left(E_{E} \otimes P_{y} \otimes\left(-A+2 \Sigma_{E} A^{-}\right)\right) U_{h} \\
& +U_{h}^{T}\left(P_{x} \otimes E_{S} \otimes\left(B+2 \Sigma_{S} B^{+}\right)\right) U_{h} \\
& +U_{h}^{T}\left(P_{x} \otimes E_{N} \otimes\left(-B+\Sigma_{N} B^{-}\right)\right) U_{h}
\end{aligned}
$$

and it is required that we chose $\Sigma_{W, E, S, N}$ so that $\left\|U_{h}\right\|_{t}^{2} \leq 0$. Since all matrices in the first two components of the Kronecker products are positive, it is sufficient to only consider the third component. Thus, we require that

$$
A+2 \Sigma_{W} A^{+} \leq 0, \quad-A+2 \Sigma_{E} A^{-} \leq 0, \quad B+2 \Sigma_{S} B^{+} \leq 0, \quad-B+\Sigma_{N} B^{-} \leq 0 .
$$

The conditions (41) are seen to be satisfied by (38) and (39) by diagonalizing $A, B$, and splitting into their positive and negative parts, respectively. Hence the scheme is energy stable if the relations in (39) hold. More details can be found in. ${ }^{22,25}$

To determine the coefficients $\sigma_{W, E, S, N}$ in (38) so that the scheme is dual consistent, we write (37), using (38), as

$$
\frac{d}{d t} U_{h}+L_{h} U_{h}=F
$$

where

$$
\begin{aligned}
L_{h} & =\left(P_{x}^{-1} Q_{x} \otimes I_{y} \otimes A\right)+\left(I_{x} \otimes P_{y}^{-1} Q_{y} \otimes B\right) \\
& -\left(P_{x}^{-1} E_{W} \otimes I_{y} \otimes \sigma_{W} A^{+}\right)-\left(P_{x}^{-1} E_{E} \otimes I_{y} \otimes \sigma_{E} A^{-}\right) \\
& -\left(I_{x} \otimes P_{y}^{-1} E_{S} \otimes \sigma_{S} B^{+}\right)-\left(I_{x} \otimes P_{y}^{-1} E_{N} \otimes \sigma_{N} B^{-}\right)
\end{aligned}
$$

and we have to compute the dual operator $L_{h}^{*}$ so that it becomes a consistent approximation of (35) with the dual boundary conditions (36). The coefficients so that (37) is dual consistent are given in

Theorem II.2. The scheme (37) is dual consistent with (38) and the choices

$$
\sigma_{W}=-1, \quad \sigma_{E}=1, \quad \sigma_{S}=-1, \quad \sigma_{N}=1 .
$$

Proof. In analogy with (14) we compute the dual operator, according to the definition

$$
L_{h}^{*}=\left(P_{x} \otimes P_{y} \otimes I_{4}\right)^{-1} L_{h}^{T}\left(P_{x} \otimes P_{y} \otimes I_{4}\right),
$$

as

$$
\begin{aligned}
L_{h}^{*} & =-\left(P_{x}^{-1} Q_{x} \otimes I_{y} \otimes A\right)-\left(I_{x} \otimes P_{y}^{-1} Q_{y} \otimes B\right) \\
& -\left(P_{x}^{-1} E_{W} \otimes I_{y} \otimes A^{-}\right)+\left(P_{x}^{-1} E_{E} \otimes I_{y} \otimes A^{+}\right) \\
& -\left(I_{x} \otimes P_{y}^{-1} E_{S} \otimes B^{-}\right)+\left(I_{x} \otimes P_{y}^{-1} E_{N} \otimes B^{+}\right) \\
& +\left(P_{x}^{-1} E_{W} \otimes I_{y} \otimes\left(1+\sigma_{W}\right) A^{+}\right)-\left(P_{x}^{-1} E_{E} \otimes I_{y} \otimes\left(1-\sigma_{E}\right) A^{-}\right) \\
& +\left(I_{x} \otimes P_{y}^{-1} E_{S} \otimes\left(1+\sigma_{S}\right) B^{+}\right)-\left(I_{x} \otimes P^{-1} E_{N} \otimes\left(1-\sigma_{N}\right) B^{-}\right) .
\end{aligned}
$$

The four last terms in (46) imposes the boundary conditions

$$
A^{+} \theta=0, \quad A^{-} \theta=0, \quad B^{+} \theta=0, \quad B^{-} \theta=0
$$

at the west, east, south and north boundaries, respectively. These are not valid boundary conditions for the dual problem and are canceled by the choices in (44). Since none of conditions contradicts the stability conditions (39), the scheme is both stable and dual consistent. 


\section{B. Numerical results for the Euler equations}

The theory of functional superconvergence is based on linear problems with constant coefficients and linear integral functionals. For these problems the theoretical and numerical results are in good agreement. $4,8,9,15,21$ Here we will apply the linear theory to the fully non-linear Euler equations in conservative form to see whether or not the theory holds also in this case.

To compute the rate of convergence, a forcing function has been added to the right-hand-side so that the solution is given by

$$
\begin{array}{ll}
\rho=1+\frac{1}{2} \sin (\pi(x-y)-t), & u=\frac{1}{2} \cos (\pi x+y-t), \\
v=\frac{1}{2} \sin (x+\pi y-t), & p=1+\frac{1}{2} \cos (\pi(x-y)-t) \sin (\pi(x+y)-t) .
\end{array}
$$

This is sometimes referred to as the method of manufactured solutions..$^{27,28}$

The addition of the forcing function does not alter the well-posedness properties or boundary conditions due to the principle of Duhamel. ${ }^{26}$ We perform a mesh refinement study from 32 to 256 grid points where the time integration is done using the classical 4th-order Runge-Kutta method until time $t=0.1$ using 1000 time steps.

To avoid showing too many results, we only show the convergence results for the 8th-order operator which results in a 5th-order accurate solution, see. ${ }^{20}$ For the Euler equations, we show the results for the both the dual consistent as in (44) discretization as well as the dual inconsistent as in (39). The rates of convergence for the conservative variables for the dual consistent case are seen in Table 1 and for the dual inconsistent case in Table 2.

Table 1. Convergence rates $q_{r}$ for the conservative variables in the Euler equations using the dual consistent discretization

\begin{tabular}{|c|c|c|c|c|}
\hline $\mathrm{N}$ & $q_{r}(\rho)$ & $q_{r}(\rho u)$ & $q_{r}(\rho v)$ & $q_{r}(e)$ \\
\hline 64 & 4.7498 & 5.1201 & 5.2513 & 5.3562 \\
96 & 5.5454 & 5.1739 & 5.6076 & 5.4327 \\
128 & 5.7281 & 5.3122 & 5.3432 & 5.4978 \\
160 & 5.4519 & 5.2569 & 4.9460 & 5.4139 \\
192 & 5.3262 & 5.1627 & 5.0112 & 5.3523 \\
224 & 5.3772 & 5.0984 & 5.0819 & 5.3462 \\
256 & 5.4024 & 5.0562 & 5.0123 & 5.3304 \\
\hline
\end{tabular}

Table 2. Convergence rates $q_{r}$ for the conservative variables in the Euler equations using the dual inconsistent discretization

\begin{tabular}{|c|c|c|c|c|}
\hline $\mathrm{N}$ & $q_{r}(\rho)$ & $q_{r}(\rho u)$ & $q_{r}(\rho v)$ & $q_{r}(e)$ \\
\hline 64 & 4.6858 & 4.8067 & 4.9053 & 4.8350 \\
96 & 5.4669 & 5.1441 & 5.3276 & 5.1247 \\
128 & 5.2475 & 5.0821 & 5.0106 & 5.1784 \\
160 & 4.8783 & 4.8968 & 4.9261 & 5.1207 \\
192 & 5.1188 & 5.0338 & 5.3047 & 5.0758 \\
224 & 5.2215 & 5.0934 & 5.1739 & 5.1222 \\
256 & 5.0817 & 5.0181 & 4.9316 & 5.1390 \\
\hline
\end{tabular}

The functionals we consider are the volume integrals of the conservative variables and the volume integral 
of the pressure and the kinetic energy,

$$
\begin{array}{lll}
J_{1}(q)=\int_{\Omega} \rho d \Omega, & J_{2}(q)=\int_{\Omega} \rho u d \Omega, & J_{3}(q)=\int_{\Omega} \rho v d \Omega, \\
J_{4}(q)=\int_{\Omega} e d \Omega, & J_{5}(q)=\int_{\Omega} p d \Omega, & J_{6}(q)=\int_{\Omega} k_{e} d \Omega,
\end{array}
$$

where

$$
p=(\gamma-1)\left(e-\frac{1}{2} \rho\left(u^{2}+v^{2}\right)\right), \quad k_{e}=\frac{1}{2} \rho\left(u^{2}+v^{2}\right),
$$

are non-linear functions of the conservative variables. These functionals can be computed analytically and the rates of convergence are measured against the exact values. The rates of convergence for the functionals are seen in Table 3 for the dual consistent case and in Table 4 for the dual inconsistent case.

Table 3. Convergence rates $q_{r}$ for the functionals from the dual consistent discretization of the Euler equations. The last row is the constant least square (cls) fit which represent an expected value.

\begin{tabular}{|c|c|c|c|c|c|c|}
\hline $\mathrm{N}$ & $q_{r}\left(J_{1}\right)$ & $q_{r}\left(J_{2}\right)$ & $q_{r}\left(J_{3}\right)$ & $q_{r}\left(J_{4}\right)$ & $q_{r}\left(J_{5}\right)$ & $q_{r}\left(J_{6}\right)$ \\
\hline 64 & 4.5780 & 5.3914 & 4.8985 & 4.5785 & 4.5495 & 4.3555 \\
96 & 8.1389 & 10.2924 & 13.2182 & 8.3594 & 8.1815 & 7.2962 \\
128 & 4.1186 & 1.3917 & -1.3703 & 4.7803 & 5.2629 & 8.4514 \\
160 & 6.6508 & 7.0548 & 11.5813 & 8.0494 & 8.0560 & 8.1333 \\
192 & 10.8908 & 8.8689 & 27.0329 & 16.7060 & 20.5837 & 7.4007 \\
224 & 8.3161 & 6.0510 & -3.6784 & 17.4289 & 2.2955 & 2.5346 \\
256 & 3.1693 & 4.1337 & 0.2751 & -5.9980 & 19.2379 & 7.1131 \\
\hline cls & 6.5518 & 6.1691 & 7.4225 & 7.7006 & 9.7381 & 6.4693 \\
\hline
\end{tabular}

Table 4. Convergence rates $q_{r}$ for the functionals from the dual inconsistent discretization of the Euler equations. The last row is the constant least square (cls) fit which represent an expected value.

\begin{tabular}{|c|c|c|c|c|c|c|}
\hline $\mathrm{N}$ & $q_{r}\left(J_{1}\right)$ & $q_{r}\left(J_{2}\right)$ & $q_{r}\left(J_{3}\right)$ & $q_{r}\left(J_{4}\right)$ & $q_{r}\left(J_{5}\right)$ & $q_{r}\left(J_{6}\right)$ \\
\hline 64 & 4.6017 & 4.9105 & 4.7553 & 4.9072 & 4.9909 & 3.8402 \\
96 & 5.0038 & 5.1344 & 5.1212 & 5.0948 & 5.1267 & 4.8317 \\
128 & 5.1127 & 5.0674 & 5.1322 & 5.0634 & 5.0657 & 5.0456 \\
160 & 4.8696 & 5.1995 & 5.0439 & 5.0918 & 5.1176 & 4.8976 \\
192 & 4.9294 & 5.2151 & 5.1612 & 5.1669 & 5.1827 & 5.0520 \\
224 & 5.1733 & 5.1164 & 5.2961 & 5.1918 & 5.1828 & 5.2568 \\
256 & 5.1197 & 5.1597 & 5.2264 & 5.1542 & 5.1542 & 5.1542 \\
\hline cls & 4.9729 & 5.1147 & 5.1052 & 5.0957 & 5.1172 & 4.8683 \\
\hline
\end{tabular}

As we can see from Tables 1 and 2, the rate of convergence for the conservative variables does not differ between the dual consistent and dual inconsistent discretization.

From Table 3 we can see that the superconvergence is not clearly visible. From linear theory, one should expect 8th-order accuracy. The rates of convergence are at any rate higher than for the dual inconsistent case in Table 4. Note the non-smooth behavior of the rates of convegence for the dual consistent case. This was seen also for linear equations ${ }^{9}$ and is due to the lack of dissipation for the PDE itself. The dual inconsistent case attains its design order 5th-order accuracy also for the functionals. 


\section{The 2-D Navier-Stokes equations}

The two-dimensional, compressible Navier-Stokes equations in non-dimensional form can be written in conservative form as

$$
q_{t}+F_{x}^{I}+G_{y}^{I}=\varepsilon\left(F_{x}^{V}+G_{y}^{V}\right),
$$

where $q, F^{I}$, and $G^{I}$ are as before. The coefficient $\varepsilon=M a / R e$ is the ratio between the Mach and Reynolds numbers and the viscous fluxes are given by

$$
\begin{aligned}
& F^{V}=\left[0, \tau_{x x}, \tau_{x y}, u \tau_{x x}+v \tau_{x y}+\kappa T_{x}\right]^{T}, \\
& G^{V}=\left[0, \tau_{x y}, \tau_{y y}, u \tau_{y x}+v \tau_{y y}+\kappa T_{y}\right]^{T},
\end{aligned}
$$

where $\kappa$ is the thermal conductivity coefficient. The stress tensor is given by

$$
\begin{aligned}
& \tau_{x x}=2 \mu \frac{\partial u}{\partial x}+\lambda\left(\frac{\partial u}{\partial x}+\frac{\partial v}{\partial y}\right), \tau_{y y}=2 \mu \frac{\partial v}{\partial y}+\lambda\left(\frac{\partial u}{\partial x}+\frac{\partial v}{\partial y}\right), \\
& \tau_{x y}=\tau_{y x}=\mu\left(\frac{\partial u}{\partial y}+\frac{\partial v}{\partial x}\right),
\end{aligned}
$$

where $\mu$ and $\lambda$ are the dynamic and second viscosity, respectively. More details can be found in i.e. ${ }^{22,25,29}$

Since (51) is a highly non-linear system of equations it cannot be analyzed using standard theory. We hence follow the procedure in section II and linearize (51) around a constant state. By applying the parabolic symmetrizer derived in ${ }^{24}$ we obtain the symmetric constant coefficient system

$$
U_{t}+A U_{x}+B U_{y}=\varepsilon\left(\left(C_{11} U_{x}+C_{12} U_{y}\right)_{x}+\left(C_{21} U_{x}+C_{22} U_{y}\right)_{y}\right),
$$

where $U, A, B$ are as before and the matrices $C_{11}, C_{12}, C_{21}$ and $C_{22}$ can again be found in. ${ }^{24,25}$

\section{A. Well-posed boundary conditions for the primal problem}

In order to derive well-posed boundary conditions for the primal problem (51), we consider the unit square and a flow going from left to right. We have linearized around a state with constant $x, y$-directional velocities $\bar{u}, \bar{v}>0$. The west/south boundaries are inflow boundaries, while the east/north boundaries are outflow boundaries. In this case, the west/south boundaries require 4 boundary conditions while the east/north boundaries require 3 boundary conditions. ${ }^{25}$ See figure 1 .

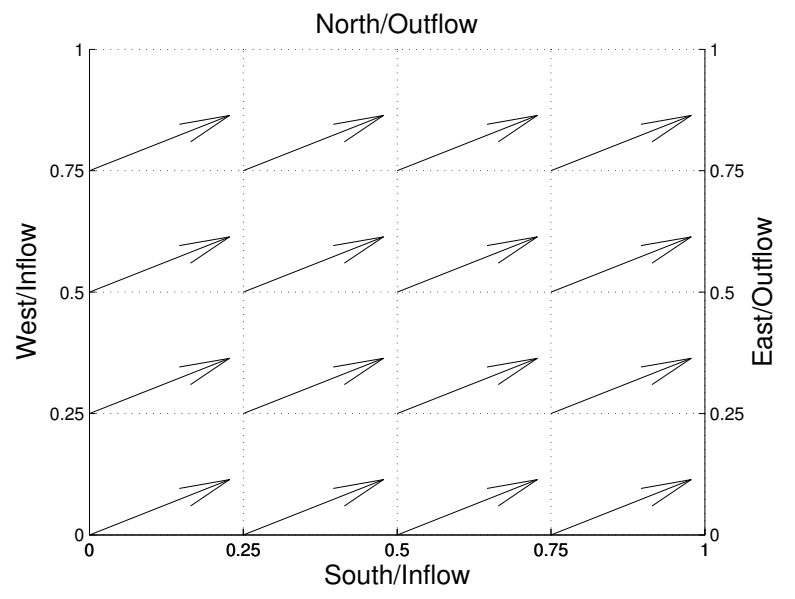

Figure 1. Computational domain and flow assumptions

The (homogeneous) boundary conditions we consider are of the form

$$
\begin{aligned}
H_{W} U-\varepsilon\left(C_{11} U_{x}+C_{12} U_{y}\right) & =0, \\
H_{E} U+\varepsilon\left(C_{11} U_{x}+C_{12} U_{y}\right) & =0, \\
H_{S} U-\varepsilon\left(C_{21} U_{x}+C_{22} U_{y}\right) & =0, \\
H_{N} U+\varepsilon\left(C_{21} U_{x}+C_{22} U_{y}\right) & =0,
\end{aligned}
$$


for the west, east, south and north boundaries, respectively. The matrices $H_{W, E, S, N}$ are yet to be determined for well-posedness of both the primal and dual problems.

A necessary, but not sufficient, requirement on $H_{W, E, S, N}$ is that they give an energy estimate. By applying the energy method to (54) and using the Green-Gauss formula for higher dimensional integration-by-parts we obtain,

$$
\begin{aligned}
\|U\|_{t}^{2} & =-\int_{0}^{1} U^{T}\left(-A+H_{W}+H_{W}^{T}\right) U d y-\int_{0}^{1} U^{T}\left(A+H_{E}+H_{E}^{T}\right) U d y \\
& -\int_{0}^{1} U^{T}\left(-B+H_{S}+H_{S}^{T}\right) U d x-\int_{0}^{1} U^{T}\left(B+H_{N}+H_{N}^{T}\right) U d x \\
& -2 \varepsilon \int_{\Omega} \nabla U^{T} \cdot\left(C_{11} U_{x}+C_{12} U_{y}, C_{21} U_{x}+C_{22} U_{y}\right) d \Omega .
\end{aligned}
$$

The last term in (56) is dissipative 22,25 and it is clear that $H_{W, E, S, N}$ must be chosen so that

$$
\begin{array}{ll}
-A+H_{W}+H_{W}^{T} \geq 0, & A+H_{E}+H_{E}^{T} \geq 0, \\
-B+H_{S}+H_{S}^{T} \geq 0, & B+H_{N}+H_{N}^{T} \geq 0,
\end{array}
$$

in order to obtain a bounded energy growth and hence an energy estimate. The specific form of $H_{W, E, S, N}$ will be determined after considering well-posedness of the dual problem.

\section{B. Well-posed boundary conditions for the dual problem}

To determine the dual problem we write (54) as

$$
U_{t}+L U=0
$$

where

$$
L=A \frac{\partial}{\partial x}+B \frac{\partial}{\partial y}-\varepsilon\left(C_{11} \frac{\partial^{2}}{\partial x^{2}}+C_{12} \frac{\partial^{2}}{\partial y x}+C_{21} \frac{\partial^{2}}{\partial x y}+C_{22} \frac{\partial^{2}}{\partial y y}\right) .
$$

By adding a forcing function, $R$, and letting $U_{t}=0$, we seek a function $\theta$ so that

$$
J(U)=(\theta, R),
$$

where

$$
J(U)=(S, U)
$$

is a linear functional of interest. Repeated use of the Green-Gauss formula results in

$$
\begin{aligned}
J(U)= & J(U)-(\theta, L U-R) \\
& =(\theta, R)+\left(S-L^{*} \theta, U\right) \\
& +\int_{0}^{1} \theta^{T}\left(A U-\varepsilon\left(C_{11} U_{x}+C_{12} U_{y}\right) d y+\varepsilon \int_{0}^{1}\left(\theta_{x}^{T} C_{11}+\theta_{y}^{T} C_{12}\right) U d y\right. \\
& -\int_{0}^{1} \theta^{T}\left(A U-\varepsilon\left(C_{11} U_{x}+C_{12} U_{y}\right) d y-\varepsilon \int_{0}^{1}\left(\theta_{x}^{T} C_{11}+\theta_{y}^{T} C_{12}\right) U d y\right. \\
& +\int_{0}^{1} \theta^{T}\left(B U-\varepsilon\left(C_{21} U_{x}+C_{22} U_{y}\right) d x+\varepsilon \int_{0}^{1}\left(\theta_{x}^{T} C_{21}+\theta_{y}^{T} C_{22}\right) U d x\right. \\
& -\int_{0}^{1} \theta^{T}\left(B U-\varepsilon\left(C_{21} U_{x}+C_{22} U_{y}\right) d x-\varepsilon \int_{0}^{1}\left(\theta_{x}^{T} C_{21}+\theta_{y}^{T} C_{22}\right) U d x .\right.
\end{aligned}
$$


The dual operator, $L^{*}$, is given by

$$
L^{*}=-A \frac{\partial}{\partial x}-B \frac{\partial}{\partial y}-\varepsilon\left(C_{11} \frac{\partial^{2}}{\partial x^{2}}+C_{12} \frac{\partial^{2}}{\partial y x}+C_{21} \frac{\partial^{2}}{\partial x y}+C_{22} \frac{\partial^{2}}{\partial y y}\right)
$$

and we obtain the dual boundary conditions by applying the homogeneous primal boundary conditions to the boundary integral terms. By using (55), we can write (62) as

$$
\begin{aligned}
J(U) & =(\theta, R)+\left(S-L^{*} \theta, U\right) \\
& +\int_{0}^{1} U^{T}\left(\left(A-H_{W}^{T}\right) \theta+\varepsilon\left(C_{11} \theta_{x}+C_{12} \theta_{y}\right)\right) d y, \\
& -\int_{0}^{1} U^{T}\left(\left(A+H_{E}^{T}\right) \theta+\varepsilon\left(C_{11} \theta_{x}+C_{12} \theta_{y}\right)\right) d y, \\
& +\int_{0}^{1} U^{T}\left(\left(B-H_{S}^{T}\right) \theta+\varepsilon\left(C_{21} \theta_{x}+C_{22} \theta_{y}\right)\right) d x, \\
& -\int_{0}^{1} U^{T}\left(\left(B+H_{N}^{T}\right) \theta+\varepsilon\left(C_{21} \theta_{x}+C_{22} \theta_{y}\right)\right) d x,
\end{aligned}
$$

and hence the dual boundary conditions are given by

$$
\begin{aligned}
\left(A-H_{W}^{T}\right) \theta+\varepsilon\left(C_{11} \theta_{x}+C_{12} \theta_{y}\right) & =0, \\
\left(A+H_{E}^{T}\right) \theta+\varepsilon\left(C_{11} \theta_{x}+C_{12} \theta_{y}\right) & =0, \\
\left(B-H_{S}^{T}\right) \theta+\varepsilon\left(C_{21} \theta_{x}+C_{22} \theta_{y}\right) & =0, \\
\left(B+H_{N}^{T}\right) \theta+\varepsilon\left(C_{21} \theta_{x}+C_{22} \theta_{y}\right) & =0,
\end{aligned}
$$

for the west, east, south and north boundaries, respectively.

Once the dual operator, $L^{*}$, and the dual boundary conditions (65) have been determined, we can consider the time-dependent dual problem. By again applying the time transformation

$$
\tau=T-t
$$

we can write the time-dependent dual problem as

$$
\theta_{\tau}-A \theta_{x}-B \theta_{y}=\varepsilon\left(\left(C_{11} \theta_{x}+C_{12} \theta_{y}\right)_{x}+\left(C_{21} \theta_{x}+C_{22} \theta_{y}\right)_{y}\right) .
$$

It is necessary, but not sufficient, that the dual boundary conditions in (65) gives an energy estimate for the time-dependent dual problem (67). The energy method applied to (67) results, as before, in

$$
\begin{aligned}
\|\theta\|_{\tau}^{2} & =-\int_{0}^{1} \theta^{T}\left(-A+H_{W}+H_{W}^{T}\right) \theta d y-\int_{0}^{1} \theta^{T}\left(A+H_{E}+H_{E}^{T}\right) \theta d y \\
& -\int_{0}^{1} \theta^{T}\left(-B+H_{S}+H_{S}^{T}\right) \theta d x-\int_{0}^{1} \theta^{T}\left(B+H_{N}+H_{N}^{T}\right) \theta d x \\
& -2 \varepsilon \int_{\Omega} \nabla \theta^{T} \cdot\left(C_{11} \theta_{x}+C_{12} \theta_{y}, C_{21} \theta_{x}+C_{22} \theta_{y}\right) d \Omega .
\end{aligned}
$$

and we can see that the same requirements for obtaining an energy estimate holds for the dual problem as for the primal problem. It is thus sufficient to construct the matrices $H_{W, E, S, N}$ so that (57) holds and so that the correct number of boundary conditions are imposed for both the primal and dual problems. An energy estimate for both problems will follow. 


\section{Well-posed boundary conditions for both the primal and dual problems}

To obtain well-posedess, it is necessary that an energy estimate is obtained with the correct number of boundary conditions. Such an operator is called maximally semi-bounded. ${ }^{26}$ Too many boundary conditions can prevent a solution from existing, and too few results in non-unique solutions. The reasoning regarding the number of boundary conditions is identical for all boundaries, and hence we restrict the attention to the west and east boundaries for simplicity.

The specific form of the matrices $H_{W, E}$ in the boundary conditions (55) and (65) can now be determined. Since the west boundary is an inflow boundary, it requires 4 boundary conditions for the primal problem. The west boundary for the dual problem, however, is an outflow boundary and only 3 boundary conditions can be used. See ${ }^{25,30}$ for details about the number of required boundary conditions. Thus, it is required that

$$
A-H_{W}^{T}=\left[\begin{array}{cccc}
0 & 0 & 0 & 0 \\
\alpha_{21} & \alpha_{22} & \alpha_{23} & \alpha_{24} \\
\alpha_{31} & \alpha_{32} & \alpha_{33} & \alpha_{34} \\
\alpha_{41} & \alpha_{42} & \alpha_{43} & \alpha_{44}
\end{array}\right]
$$

or equivalently,

$$
H_{W}=\left[\begin{array}{cccc}
\bar{u} & \frac{\bar{c}}{\sqrt{\gamma}}-\alpha_{21} & -\alpha_{31} & -\alpha_{41} \\
\bar{c} & \bar{u}-\alpha_{22} & -\alpha_{32} & \bar{c} \sqrt{\frac{\gamma-1}{\gamma}}-\alpha_{42} \\
0 & -\alpha_{23} & \bar{u}-\alpha_{33} & -\alpha_{43} \\
0 & \bar{c} \sqrt{\frac{\gamma-1}{\gamma}-\alpha_{24}} & -\alpha_{34} & \bar{u}-\alpha_{44}
\end{array}\right] .
$$

Unless $H_{W}$ have this form, the top row of $A-H_{W}^{T}$ is non-zero and too many boundary conditions would be imposed for the dual problem, making it ill-posed since a solution to overdetermined problems might not exist. We can also see that the top row of $H_{W}$ is always non-zero and thus there are 4 boundary conditions imposed for the primal problem at all times.

We can apply the same reasoning for the east boundary. The east boundary is an outflow boundary for the primal problem and hence 3 boundary conditions are required. For the dual problem, however, we are allowed to use 4 boundary conditions. This immediately puts restrictions on $H_{E}$ to have the form

$$
H_{E}=\left[\begin{array}{cccc}
0 & 0 & 0 & 0 \\
\beta_{21} & \beta_{22} & \beta_{23} & \beta_{24} \\
\beta_{31} & \beta_{32} & \beta_{33} & \beta_{34} \\
\beta_{41} & \beta_{42} & \beta_{43} & \beta_{44}
\end{array}\right]
$$

or too many boundary conditions would be imposed for the primal problem, making it ill-posed. Since the dual problem requires 4 boundary conditions, it is also required that the top row of $A+H_{E}^{T}$ is non-zero. By computing

$$
A+H_{E}^{T}=\left[\begin{array}{cccc}
\bar{u} & \frac{\bar{c}}{\sqrt{\gamma}}+\beta_{21} & \beta_{31} & \beta_{41} \\
\bar{c} & \bar{u}+\beta_{22} & \beta_{32} & \bar{c} \sqrt{\frac{\gamma-1}{\gamma}}+\beta_{42} \\
0 & \beta_{23} & \bar{u}+\beta_{33} & \beta_{43} \\
0 & \bar{c} \sqrt{\frac{\gamma-1}{\gamma}}+\beta_{24} & \beta_{34} & \bar{u}+\beta_{44}
\end{array}\right],
$$

we can see that the top left entry will be non-zero and hence 4 boundary conditions for the dual problem will be imposed at all times.

The same reasoning can be applied to the south/north boundaries which gives the form of the matrices 


$$
\begin{aligned}
H_{S} & =\left[\begin{array}{cccc}
\bar{v} & -\zeta_{21} & \frac{\bar{c}}{\sqrt{\gamma}}-\zeta_{31} & -\zeta_{41} \\
0 & \bar{v}-\zeta_{22} & -\zeta_{32} & -\zeta_{42} \\
\bar{c} & -\zeta_{23} & \bar{v}-\zeta_{33} & \bar{c} \sqrt{\frac{\gamma-1}{\gamma}-\zeta_{43}} \\
\frac{\sqrt{\gamma}}{0} & -\zeta_{24} & \bar{c} \sqrt{\frac{\gamma-1}{\gamma}}-\zeta_{34} & \bar{v}-\zeta_{44}
\end{array}\right], \\
H_{N} & =\left[\begin{array}{cccc}
0 & 0 & 0 & 0 \\
\eta_{21} & \eta_{22} & \eta_{23} & \eta_{24} \\
\eta_{31} & \eta_{32} & \eta_{33} & \eta_{34} \\
\eta_{41} & \eta_{42} & \eta_{43} & \eta_{44}
\end{array}\right] .
\end{aligned}
$$

Once that the form of the matrices $H_{W, E, S, N}$ have been determined, it is a matter of choosing the coefficients $\alpha_{i} \in H_{W}, \beta_{j} \in H_{E}, \zeta_{k} \in H_{S}$ and $\eta_{l} \in H_{N}$ so that (57) hold. The choice of coefficients are not unique and there are plenty of combinations which satisfy the requirements. One particular choice is given in

Theorem III.1. Both the primal problem (54) with the primal boundary conditions (55) and the dual problem (67) with the dual boundary conditions (65), are well-posed with the choice of the coefficients $\alpha_{i} \in H_{W}$, $\beta_{j} \in H_{E}, \zeta_{k} \in H_{S}$ and $\eta_{l} \in H_{N}$ given by

$$
\begin{aligned}
& \alpha_{21}=\frac{\bar{c}}{\sqrt{\gamma}}, \quad \beta_{21}=-\frac{\bar{c}}{\sqrt{\gamma}}, \quad \zeta_{21}=0, \quad \eta_{21}=0, \\
& \alpha_{31}=0, \quad \beta_{31}=0, \quad \zeta_{31}=\frac{\bar{c}}{\sqrt{\gamma}}, \quad \eta_{31}=-\frac{\bar{c}}{\sqrt{\gamma}}, \\
& \alpha_{41}=0, \quad \beta_{41}=0, \quad \zeta_{41}=0, \quad \eta_{41}=0, \\
& \alpha_{32}=-\alpha_{23}, \quad \beta_{32}=-\beta_{23}, \quad \zeta_{32}=-\zeta_{23}, \quad \eta_{32}=-\eta_{23}, \\
& \alpha_{42}=-\alpha_{24}+\bar{c} \sqrt{\frac{\gamma-1}{\gamma}}, \quad \beta_{42}=-\beta_{24}-\bar{c} \sqrt{\frac{\gamma-1}{\gamma}}, \quad \zeta_{42}=-\zeta_{24}, \quad \eta_{42}=-\eta_{24}, \\
& \alpha_{43}=-\alpha_{34}, \quad \beta_{43}=-\beta_{34}, \quad \zeta_{43}=\bar{c} \sqrt{\frac{\gamma-1}{\gamma}}-\zeta_{34}, \quad \eta_{43}=-\bar{c} \sqrt{\frac{\gamma-1}{\gamma}}-\eta_{34} \text {, } \\
& \alpha_{22} \leq \frac{\bar{u}}{2}, \quad \beta_{22} \geq-\frac{\bar{u}}{2}, \quad \zeta_{22} \leq \frac{\bar{v}}{2}, \quad \eta_{22} \geq-\frac{\bar{v}}{2}, \\
& \alpha_{33} \leq \frac{\bar{u}}{2}, \quad \beta_{33} \geq-\frac{\bar{u}}{2}, \quad \zeta_{33} \leq \frac{\bar{v}}{2}, \quad \eta_{33} \geq-\frac{\bar{v}}{2}, \\
& \alpha_{44} \leq \frac{\bar{u}}{2}, \quad \beta_{44} \geq-\frac{\bar{u}}{2} \quad \zeta_{44} \leq \frac{\bar{v}}{2}, \quad \eta_{44} \geq-\frac{\bar{v}}{2} \text {. }
\end{aligned}
$$

Proof. The matrices $H_{W, E, S, N}$ are constructed to give the correct number of boundary conditions for the primal and dual problems. We need to make sure that the relations in (74) are sufficient to obtain energy estimates. For both the primal and dual problems, it it sufficient that the coefficients in (74) ensures that 
(57) holds. By inserting the relations with equality into (57) we obtain

$$
\begin{aligned}
-A+H_{W}+H_{W}^{T} & =\left[\begin{array}{cccc}
\bar{u} & 0 & 0 & 0 \\
0 & \bar{u}-2 \alpha_{22} & 0 & 0 \\
0 & 0 & \bar{u}-2 \alpha_{33} & 0 \\
0 & 0 & 0 & \bar{u}-2 \alpha_{44}
\end{array}\right], \\
A+H_{E}+H_{E}^{T} & =\left[\begin{array}{cccc}
\bar{u} & 0 & 0 & 0 \\
0 & \bar{u}+2 \beta_{22} & 0 & 0 \\
0 & 0 & \bar{u}+2 \beta_{33} & 0 \\
0 & 0 & 0 & \bar{u}+2 \beta_{44}
\end{array}\right], \\
-B+H_{S}+H_{S}^{T} & =\left[\begin{array}{cccc}
\bar{v} & 0 & 0 & 0 \\
0 & \bar{v}-2 \zeta_{22} & 0 & 0 \\
0 & 0 & \bar{v}-2 \zeta_{33} & 0 \\
0 & 0 & 0 & \bar{v}-2 \zeta_{44}
\end{array}\right], \\
B+H_{N}+H_{N}^{T} & =\left[\begin{array}{cccc}
\bar{v} & 0 & 0 & 0 \\
0 & \bar{v}+2 \eta_{22} & 0 & 0 \\
0 & 0 & \bar{v}+2 \eta_{33} & 0 \\
0 & 0 & 0 & \bar{v}+2 \eta_{44}
\end{array}\right],
\end{aligned}
$$

and the inequality relations ensures that

$$
\begin{array}{ll}
-A+H_{W}+H_{W}^{T} \geq 0, & A+H_{E}+H_{E}^{T} \geq 0, \\
-B+H_{S}+H_{S}^{T} \geq 0, & B+H_{N}+H_{N}^{T} \geq 0 .
\end{array}
$$

Hence we impose the correct number of boundary conditions for both the primal and dual problems, and energy estimates are obtained.

Remark III.1. Note that there are many possible choices of the coefficients in $H_{W, E, S, N}$ so that (57) holds. Theorem III.1 is merely one choice with the purpose of making the matrices in the energy estimates diagonal. Even so, there are several free parameters which can be chosen arbitrarily and used for various optimization purposes. For example, the coefficients can be chosen so that the boundary conditions for the primal and dual Navier-Stokes equation converge to well-posed boundary conditions for the primal and dual Euler equations as $\varepsilon \rightarrow 0$. This is ongoing work and will be presented in a future paper. With the current choices, there is no convergence to the Euler equations as $\varepsilon \rightarrow 0$.

\section{Discretization, stability, and dual consistency}

To perform a stability analysis, we discretize the linear and symmetric system (54) with the boundary

conditions in (55). In the computations, however, the non-linear equations (51) is used and the system have been transformed to its conservative form.

An SBP-SAT discretization of (54) can be written as

$$
\begin{aligned}
& \frac{d}{d t} U_{h}+\left(P_{x}^{-1} Q_{x} \otimes I_{y} \otimes A\right) U_{h}+\left(I_{x} \otimes P_{y}^{-1} Q_{y} \otimes B\right) U_{h} \\
& -\varepsilon\left(P_{x}^{-1} Q_{x} P_{x}^{-1} Q_{x} \otimes I_{y} \otimes C_{11}\right) U_{h}-\varepsilon\left(P_{x}^{-1} Q_{x} \otimes P_{y}^{-1} Q_{y} \otimes C_{12}\right) U_{h} \\
& -\varepsilon\left(P_{x}^{-1} Q_{x} \otimes P_{y}^{-1} Q_{y} \otimes C_{21}\right) U_{h}-\varepsilon\left(I_{x} \otimes P_{y}^{-1} Q_{y} P_{y}^{-1} Q_{y} \otimes C_{22}\right) U_{h} \\
& =\left(P^{-1} E_{W} \otimes I_{y} \otimes \Sigma_{W}\right)\left(\left(I_{x} \otimes I_{y} \otimes H_{W}\right) U_{h}-\varepsilon\left(\left(P_{x}^{-1} Q_{x} \otimes I_{y} \otimes C_{11}\right) U_{h}+\left(I_{x} \otimes P_{y}^{-1} Q_{y} \otimes C_{12}\right) U_{h}\right)\right) \\
& +\left(P^{-1} E_{E} \otimes I_{y} \otimes \Sigma_{E}\right)\left(\left(I_{x} \otimes I_{y} \otimes H_{E}\right) U_{h}+\varepsilon\left(\left(P_{x}^{-1} Q_{x} \otimes I_{y} \otimes C_{11}\right) U_{h}+\left(I_{x} \otimes P_{y}^{-1} Q_{y} \otimes C_{12}\right) U_{h}\right)\right) \\
& +\left(I_{x} \otimes P_{y}^{-1} E_{S} \otimes \Sigma_{S}\right)\left(\left(I_{x} \otimes I_{y} \otimes H_{S}\right) U_{h}-\varepsilon\left(\left(P_{x}^{-1} Q_{x} \otimes I_{y} \otimes C_{21}\right) U_{h}+\left(I_{x} \otimes P_{y}^{-1} Q_{y} \otimes C_{22}\right) U_{h}\right)\right) \\
& +\left(I_{x} \otimes P_{y}^{-1} E_{N} \otimes \Sigma_{N}\right)\left(\left(I_{x} \otimes I_{y} \otimes H_{N}\right) U_{h}+\varepsilon\left(\left(P_{x}^{-1} Q_{x} \otimes I_{y} \otimes C_{21}\right) U_{h}+\left(I_{x} \otimes P_{y}^{-1} Q_{y} \otimes C_{22}\right) U_{h}\right)\right)
\end{aligned}
$$


where the terms before the equality sign approximate the equations, and the terms after imposes the boundary conditions (55). The matrices $\Sigma_{W, E, S, N}$ are given in

Theorem IV.1. The scheme (77) is energy stable when chosing

$$
\Sigma_{W}=\Sigma_{E}=\Sigma_{S}=\Sigma_{N}=-I_{4}
$$

where $I_{4}$ is the $4 \times 4$ identity matrix.

Proof. The energy method applied to (77) results in

$$
\begin{aligned}
\left\|U_{h}\right\|_{t}^{2} & =U_{h}^{T}\left(E_{W} \otimes P_{y} \otimes\left(A+\Sigma_{W} H_{W}+H_{W}^{T} \Sigma_{W}^{T}\right)\right) U_{h} \\
& +U_{h}^{T}\left(E_{E} \otimes P_{y} \otimes\left(-A+\Sigma_{E} H_{E}+H_{E}^{T} \Sigma_{E}^{T}\right)\right) U_{h} \\
& +U_{h}^{T}\left(P_{x} \otimes E_{S} \otimes\left(B+\Sigma_{S} H_{S}+H_{S}^{T} \Sigma_{S}^{T}\right)\right) U_{h} \\
& +U_{h}^{T}\left(P_{x} \otimes E_{N} \otimes\left(-B+\Sigma_{N} H_{N}+H_{N}^{T} \Sigma_{N}^{T}\right)\right) U_{h} \\
& -2 \varepsilon U_{h}^{T}\left(E_{W} P_{x}^{-1} Q_{x} \otimes P_{y} \otimes\left(C_{11}+\Sigma_{W} C_{11}\right)\right) U_{h} \\
& +2 \varepsilon U_{h}^{T}\left(E_{E} P_{x}^{-1} Q_{x} \otimes P_{y} \otimes\left(C_{11}+\Sigma_{W} C_{11}\right)\right) U_{h} \\
& -2 \varepsilon U_{h}^{T}\left(E_{W} \otimes Q_{y} \otimes\left(C_{12}+\Sigma_{E} C_{12}\right)\right) U_{h} \\
& +2 \varepsilon U_{h}^{T}\left(E_{E} \otimes Q_{y} \otimes\left(C_{12}+\Sigma_{E} C_{12}\right)\right) U_{h} \\
& -2 \varepsilon U_{h}^{T}\left(Q_{x} \otimes E_{S} \otimes\left(C_{21}+\Sigma_{S} C_{21}\right)\right) U_{h} \\
& +2 \varepsilon U_{h}^{T}\left(Q_{x} \otimes E_{N} \otimes\left(C_{21}+\Sigma_{N} C_{21}\right)\right) U_{h} \\
& -2 \varepsilon U_{h}^{T}\left(P_{x} \otimes E_{S} P_{y}^{-1} Q_{y} \otimes\left(C_{22}+\Sigma_{S} C_{22}\right)\right) U_{h} \\
& +2 \varepsilon U_{h}^{T}\left(P_{x} \otimes E_{N} P_{y}^{-1} Q_{y} \otimes\left(C_{22}+\Sigma_{N} C_{22}\right)\right) U_{h} \\
& -\mathrm{DI} .
\end{aligned}
$$

The last term, DI, can be written as

$$
\mathrm{DI}=\varepsilon\left[\begin{array}{c}
\left(P_{x}^{-1} Q_{x} \otimes I_{y} \otimes I_{4}\right) U \\
\left(I_{x} \otimes P_{y}^{-1} Q_{y} \otimes I_{4}\right) U
\end{array}\right]^{T}\left[\begin{array}{cc}
\left(P_{x} \otimes P_{y} \otimes C_{11}\right) & \left(P_{x} \otimes P_{y} \otimes C_{12}\right) \\
\left(P_{x} \otimes P_{y} \otimes C_{21}\right) & \left(P_{x} \otimes P_{y} \otimes C_{22}\right)
\end{array}\right]\left[\begin{array}{c}
\left(P_{x}^{-1} Q_{x} \otimes I_{y} \otimes I_{4}\right) U \\
\left(I_{x} \otimes P_{y}^{-1} Q_{y} \otimes I_{4}\right) U
\end{array}\right]
$$

and is a purely dissipative term. ${ }^{22,25}$ By the choices in (78), the expression (79) simplifies to

$$
\begin{aligned}
\left\|U_{h}\right\|_{t}^{2} & \leq-U_{h}^{T}\left(E_{W} \otimes P_{y} \otimes\left(-A+H_{W}+H_{W}^{T}\right)\right) U_{h} \\
& -U_{h}^{T}\left(E_{E} \otimes P_{y} \otimes\left(A+H_{E}+H_{E}^{T}\right)\right) U_{h} \\
& -U_{h}^{T}\left(P_{x} \otimes E_{S} \otimes\left(-B+H_{S}+H_{S}^{T}\right)\right) U_{h} \\
& -U_{h}^{T}\left(P_{x} \otimes E_{N} \otimes\left(B+H_{N}+H_{N}^{T}\right)\right) U_{h},
\end{aligned}
$$

where the matrices $H_{W, E, S, N}$ are constructed so that (57) holds and hence

$$
\left\|U_{h}\right\|_{t}^{2} \leq 0 .
$$

Thus an energy estimate have been obtained and the scheme (77) is energy stable.

In contrast to the discretization of the Euler equations, there is no freedom in the choice of penalty coefficients to obtain an energy estimate. In the Euler case, all coefficients were bounded from above or below in order to obtain an energy estimate. The requirement that the scheme should be dual consistent fixed all coefficients to unique values. In this case, all penalty coefficients are uniquely determined from the energy estimate, and it is necessary that the scheme (77) is dual consistent with the choice in (78).

To be dual consistent, it is required that the discrete operator approximates the dual operator (63) with the dual boundary conditions in (65). The main result of this paper can then be summarized in

Theorem IV.2. The discretization (77) is energy stable and dual consistent with the choice of penalty coefficients given in (78). 
Proof. Energy stability has already been proven, and we must show that the scheme (77) together with the coefficients in (78) is dual consistent. To prove this, we rewrite (77), using (78), as

$$
\frac{d}{d t} U_{h}+L_{h} U_{h}=0
$$

where

$$
\begin{aligned}
& L_{h}=\left(P_{x}^{-1} Q_{x} \otimes I_{y} \otimes A\right)+\left(I_{x} \otimes P_{y}^{-1} Q_{y} \otimes B\right) \\
& -\varepsilon\left(P_{x}^{-1} Q_{x} P_{x}^{-1} Q_{x} \otimes I_{y} \otimes C_{11}\right)-\varepsilon\left(P_{x}^{-1} Q_{x} \otimes P_{y}^{-1} Q_{y} \otimes C_{12}\right) \\
& -\varepsilon\left(P_{x}^{-1} Q_{x} \otimes P_{y}^{-1} Q_{y} \otimes C_{21}\right)-\varepsilon\left(I_{x} \otimes P_{y}^{-1} Q_{y} P_{y}^{-1} Q_{y} \otimes C_{22}\right) \\
& +\left(P^{-1} E_{W} \otimes I_{y} \otimes I_{4}\right)\left(\left(I_{x} \otimes I_{y} \otimes H_{W}\right)-\varepsilon\left(\left(P_{x}^{-1} Q_{x} \otimes I_{y} \otimes C_{11}\right)+\left(I_{x} \otimes P_{y}^{-1} Q_{y} \otimes C_{12}\right)\right)\right) \\
& +\left(P^{-1} E_{E} \otimes I_{y} \otimes I_{4}\right)\left(\left(I_{x} \otimes I_{y} \otimes H_{E}\right)+\varepsilon\left(\left(P_{x}^{-1} Q_{x} \otimes I_{y} \otimes C_{11}\right)+\left(I_{x} \otimes P_{y}^{-1} Q_{y} \otimes C_{12}\right)\right)\right) \\
& +\left(I_{x} \otimes P_{y}^{-1} E_{S} \otimes I_{4}\right)\left(\left(I_{x} \otimes I_{y} \otimes H_{S}\right)-\varepsilon\left(\left(P_{x}^{-1} Q_{x} \otimes I_{y} \otimes C_{21}\right)+\left(I_{x} \otimes P_{y}^{-1} Q_{y} \otimes C_{22}\right)\right)\right) \\
& +\left(I_{x} \otimes P_{y}^{-1} E_{N} \otimes I_{4}\right)\left(\left(I_{x} \otimes I_{y} \otimes H_{N}\right)+\varepsilon\left(\left(P_{x}^{-1} Q_{x} \otimes I_{y} \otimes C_{21}\right)+\left(I_{x} \otimes P_{y}^{-1} Q_{y} \otimes C_{22}\right)\right)\right) .
\end{aligned}
$$

The discrete dual operator can be computed according to the definition,

$$
L_{h}^{*}=\left(P_{x} \otimes P_{y} \otimes I_{4}\right)^{-1} L_{h}^{T}\left(P_{x} \otimes P_{y} \otimes I_{4}\right),
$$

as

$$
\begin{aligned}
L_{h}^{*} & =-\left(P_{x}^{-1} Q_{x} \otimes I_{y} \otimes A\right)-\left(I_{x} \otimes P_{y}^{-1} Q_{y} \otimes B\right) \\
& -\varepsilon\left(P_{x}^{-1} Q_{x} P_{x}^{-1} Q_{x} \otimes I_{y} \otimes C_{11}\right)-\varepsilon\left(P_{x}^{-1} Q_{x} \otimes P_{y}^{-1} Q_{y} \otimes C_{12}\right) \\
& -\varepsilon\left(P_{x}^{-1} Q_{x} \otimes P_{y}^{-1} Q_{y} \otimes C_{21}\right)-\varepsilon\left(I_{x} \otimes P_{y}^{-1} Q_{y} P_{y}^{-1} Q_{y} \otimes C_{22}\right) \\
& -\left(P_{x}^{-1} E_{W} \otimes I_{y} \otimes I_{4}\right)\left(\left(I_{x} \otimes I_{y} \otimes\left(A-H_{W}^{T}\right)\right)+\varepsilon\left(\left(P_{x}^{-1} Q_{x} \otimes I_{y} \otimes C_{11}\right)+\left(I_{x} \otimes P_{y}^{-1} Q_{y} \otimes C_{12}\right)\right)\right) \\
& +\left(P_{x}^{-1} E_{E} \otimes I_{y} \otimes I_{4}\right)\left(\left(I_{x} \otimes I_{y} \otimes\left(A+H_{E}^{T}\right)\right)+\varepsilon\left(\left(P_{x}^{-1} Q_{x} \otimes I_{y} \otimes C_{11}\right)+\left(I_{x} \otimes P_{y}^{-1} Q_{y} \otimes C_{12}\right)\right)\right) \\
& -\left(I_{x} \otimes P_{y}^{-1} E_{S} \otimes I_{4}\right)\left(\left(I_{x} \otimes I_{y} \otimes\left(B-H_{S}^{T}\right)\right)+\varepsilon\left(\left(P_{x}^{-1} Q_{x} \otimes I_{y} \otimes C_{21}\right)+\left(I_{x} \otimes P_{y}^{-1} Q_{y} \otimes C_{22}\right)\right)\right) \\
& +\left(I_{x} \otimes P_{y}^{-1} E_{N} \otimes I_{4}\right)\left(\left(I_{x} \otimes I_{y} \otimes\left(B+H_{N}^{T}\right)\right)+\varepsilon\left(\left(P_{x}^{-1} Q_{x} \otimes I_{y} \otimes C_{21}\right)+\left(I_{x} \otimes P_{y}^{-1} Q_{y} \otimes C_{22}\right)\right)\right) .
\end{aligned}
$$

We can see that the six first terms in (86) approximates the continuous dual operator (63), while the last four terms imposes the dual boundary conditions in (65). The discrete dual operator is thus a consistent approximation of the dual problem and the scheme (77) is hence dual consistent with the choices in (78).

Remark IV.1. Remember that the primal and dual equations have the same energy estimate in the continuous case. This holds also for the discretized equations. The energy method applied to the time-dependent discrete dual problem,

$$
\frac{d}{d \tau} \theta_{h}+L_{h}^{*} \theta=0,
$$

results in

$$
\begin{aligned}
\left\|\theta_{h}\right\|_{t}^{2} & \leq-\theta_{h}^{T}\left(E_{W} \otimes P_{y} \otimes\left(-A+H_{W}+H_{W}^{T}\right)\right) \theta_{h} \\
& -\theta_{h}^{T}\left(E_{E} \otimes P_{y} \otimes\left(A+H_{E}+H_{E}^{T}\right)\right) \theta_{h} \\
& -\theta_{h}^{T}\left(P_{x} \otimes E_{S} \otimes\left(-B+H_{S}+H_{S}^{T}\right)\right) \theta_{h} \\
& -\theta_{h}^{T}\left(P_{x} \otimes E_{N} \otimes\left(B+H_{N}+H_{N}^{T}\right)\right) \theta_{h},
\end{aligned}
$$

which is identical to the energy estimate of the discrete primal problem (81). Hence the discretization of the dual problem is also energy stable. This can open for a efficient method for simultaneous solution of the dual problem since much of the structure for the primal problem can be re-used. 


\section{A. Numerical results for the Navier-Stokes equations}

To verify the rate of convergence, we use the analytical solution given in (48) with the same parameter values. In Table 5 we show the rates of convergence for the conservative variables and in Table 6 the rates of convergence for the functionals.

The boundary conditions and numerical scheme were derived with the purpose of being dual consistent, and hence there is no dual inconsistent case to compare with. Note that we are solving the non-linear compressible Navier-Stokes equations in conservative form, and not the linear symmetric form on which the analysis have been performed.

Table 5. Convergence rates $q_{r}$ for the conservative variables in the Navier-Stokes equations

\begin{tabular}{|c|c|c|c|c|}
\hline $\mathrm{N}$ & $q_{r}(\rho)$ & $q_{r}(\rho u)$ & $q_{r}(\rho v)$ & $q_{r}(e)$ \\
\hline 64 & 4.7839 & 4.9275 & 4.7768 & 4.5232 \\
96 & 5.0482 & 4.9936 & 4.8054 & 4.5468 \\
128 & 4.9215 & 4.8725 & 4.7662 & 4.6401 \\
160 & 4.8173 & 4.8208 & 4.7526 & 4.6761 \\
192 & 4.7551 & 4.8064 & 4.7499 & 4.6957 \\
224 & 4.7162 & 4.8060 & 4.7544 & 4.7110 \\
256 & 4.6879 & 4.8116 & 4.7635 & 4.7253 \\
\hline
\end{tabular}

Table 6. Convergence rates $q_{r}$ for the functionals from the Navier-Stokes equations

\begin{tabular}{|c|c|c|c|c|c|c|}
\hline $\mathrm{N}$ & $q_{r}\left(J_{1}\right)$ & $q_{r}\left(J_{2}\right)$ & $q_{r}\left(J_{3}\right)$ & $q_{r}\left(J_{4}\right)$ & $q_{r}\left(J_{5}\right)$ & $q_{r}\left(J_{6}\right)$ \\
\hline 64 & 4.3153 & 5.5219 & 6.3834 & 1.8565 & 1.5444 & 4.9082 \\
96 & 6.1506 & 6.6984 & 7.3771 & 6.1298 & 6.0771 & 6.3547 \\
128 & 6.8749 & 7.1829 & 7.9124 & 6.8258 & 6.8054 & 6.9197 \\
160 & 7.2215 & 7.4752 & 8.2180 & 7.2135 & 7.2010 & 7.2724 \\
192 & 7.4742 & 7.6864 & 8.4029 & 7.4843 & 7.4751 & 7.5280 \\
224 & 7.6595 & 7.8422 & 8.5396 & 7.6796 & 7.6726 & 7.7142 \\
256 & 7.7906 & 7.9598 & 8.6587 & 7.8240 & 7.8135 & 7.8521 \\
\hline
\end{tabular}

We can see from Table 5 and 6 that the 5th-order accuracy for the conservative variables are almost attained, and that the functionals become superconvergence with 8th-order accuracy. Note that the rates of convergence for the functionals are much smoother than for the Euler equations, probably due to the dissipation from the PDE itself.

\section{B. A remark on the implementation}

In the derivations, the assumption has been that $\bar{u}, \bar{v} \geq 0$. If $\bar{u}<0$ or $\bar{v}<0$, the inflow boundary is changed to an outflow boundary. Since the number of boundary conditions change, the matrices are then constructed so that

$$
\begin{aligned}
& H_{W}=\left[\begin{array}{cccc}
0 & 0 & 0 & 0 \\
\alpha_{21} & \alpha_{22} & \alpha_{23} & \alpha_{24} \\
\alpha_{31} & \alpha_{32} & \alpha_{33} & \alpha_{34} \\
\alpha_{41} & \alpha_{42} & \alpha_{43} & \alpha_{44}
\end{array}\right], A+H_{E}^{T}=\left[\begin{array}{cccc}
0 & 0 & 0 & 0 \\
\beta_{21} & \beta_{22} & \beta_{23} & \beta_{24} \\
\beta_{31} & \beta_{32} & \beta_{33} & \beta_{34} \\
\beta_{41} & \beta_{42} & \beta_{43} & \beta_{44}
\end{array}\right], \\
& H_{S}=\left[\begin{array}{cccc}
0 & 0 & 0 & 0 \\
\zeta_{21} & \zeta_{22} & \zeta_{23} & \zeta_{24} \\
\zeta_{31} & \zeta_{32} & \zeta_{33} & \zeta_{34} \\
\zeta_{41} & \zeta_{42} & \zeta_{43} & \zeta_{44}
\end{array}\right], \quad B+H_{N}^{T}=\left[\begin{array}{cccc}
0 & 0 & 0 & 0 \\
\eta_{21} & \eta_{22} & \eta_{23} & \eta_{24} \\
\eta_{31} & \eta_{32} & \eta_{33} & \eta_{34} \\
\eta_{41} & \eta_{42} & \eta_{43} & \eta_{44}
\end{array}\right]
\end{aligned}
$$


where the coefficients in theorem III.1 are valid with all signs reversed. From an implementational point of view, all inequalities can be replaced by equalities for an energy stable scheme independently whether or not $\bar{u}<0$ or $\bar{v}<0$.

The stability and dual consistency theory have been derived for the linearized and symmetrized NavierStokes equations (54). In an implementation, however, the non-linear conservative flux formulation (51) is used. The stability theory can be seen as constructing a scheme for which one grid point on the boundary does not contribute to unbounded energy growth during one explicit time step, or time integration stage if a multistage method is used. In the non-linear formulation, the boundary conditions have to be applied on a nodal basis and the penalty matrices has thus to be recomputed for each node on the boundary, in each time integration stage.

One can identify terms which are common for both the non-linear and linear, symmetric formulation. For example, the boundary condition

$$
H_{W} U-\varepsilon\left(C_{11} U_{x}+C_{12} U_{y}\right)=0
$$

can be written as

$$
H_{W}(A) U-\varepsilon F_{S}^{V}=0
$$

where $F_{S}^{V}=C_{11} U_{x}+C_{12} U_{y}$ is the viscous flux and $H_{W}=H_{W}(A)$ is a function of $A$, which is the symmetrized inviscid flux jacobian. In the non-linear formulation, the viscous flux terms are always computed and the values along the boundary can be re-used in the boundary conditions. The transformation of $H_{W}$ to conservative form is done in three steps;

1. Construct $H_{W}$ from the coefficients in Theorem III.1.

2. Transform back to primitive form from the symmetric form, $H_{W}^{(P)}=S_{P} H_{W} S_{P}^{-1}$.

3. Transform back to conservative form from the primitive form, $H_{W}^{(C)}=S_{C} H_{W}^{(P)} S_{C}^{-1}$.

The transformation matrices $S_{P}$ and $S_{C}$ are found in ${ }^{24}$ and, ${ }^{31}$ respectively. Since the transformation matrices, and their inverses, are explicitly available, step 2 and 3 can be combined to a single transformation to reduce the computational complexity. Finally, the boundary condition is applied to each grid point on the boundary, in each time integration stage, as

$$
H_{W}^{(C)} q-\varepsilon F^{V}=g
$$

where $F^{V}$ is the viscous flux and $g$ is the boundary data.

\section{Conclusions}

We have derived new far-field boundary conditions for the Navier-Stokes equations. The derivations were made by simultaneously considering the primal and dual problems. The boundary conditions lead to a stable and dual consistent discretization which produced superconvergent linear and non-linear integral functionals. This superconvergence property has previously been shown for linear problems, but is now verified for non-linear problems with non-linear integral functionals.

For the Euler equations, we showed that the characteristic boundary conditions can be used to construct a stable and dual consistent discretization. Due to the lack of dissipation for the PDE itself, the superconvergence was not as clearly seen, but it was verified that the rates of convergence were higher than for a dual inconsistent discretization.

\section{Acknowledgements}

This work has partly been carried out within the IDIHOM project which is supported by the European Commission under contract No. ACP0-GA-2010-265780. Partial support was also provided by the Swedish Royal Academy of Sciences. 


\section{References}

${ }^{1}$ Jameson, A., "Aerodynamic design via control theory," Journal of Scientific Computing, Vol. 3, 1988, pp. 233-260.

${ }^{2}$ Giles, M. B. and Pierce, N. A., "An Introduction to the Adjoint Approach to Design," Flow, Turbulence and Combustion, Vol. 65, 2000, pp. 393-415.

${ }^{3}$ Pierce, N. A. and Giles, M. B., "Adjoint and defect error bounding and correction for functional estimates," Journal of Computational Physics, Vol. 200, 2004, pp. 769-794.

${ }^{4}$ Hicken, J. E., "Output error estimation for summation-by-parts finite-difference schemes," Journal of Computational Physics, Vol. 231, No. 9, 2012, pp. 3828-3848.

${ }^{5}$ Giles, M. B., Larson, M. G., Levenstam, J. M., and Süli, E., "Adaptive Error Control for Finite Element Approximations of the Lift and Drag Coefficients in Viscous Flow," Tech. rep., Report NA-97/06, Oxford University Computing Laboratory, 1997.

${ }^{6}$ Giles, M. B. and Pierce, N. A., "Superconvergent lift estimates through adjoint error analysis," Innovative Methods for Numerical Solutions of Partial Differential Equations, 2001.

${ }^{7}$ Pierce, N. A. and Giles, M. B., "Adjoint Recovery of Superconvergent Functionals from PDE Approximations," SIAM Review, Vol. 42, No. 2, 2000, pp. 247-264.

${ }^{8}$ Hicken, J. E. and Zingg, D. W., "Superconvergent Functional Estimates from Summation-By-Parts Finite-Difference Discretizations," SIAM Journal on Scientific Computing, Vol. 33, No. 2, 2011, pp. 893-922.

${ }^{9}$ Berg, J. and Nordström, J., "Superconvergent functional output for time-dependent problems using finite differences on summation-by-parts form," Journal of Computational Physics, Vol. 231, No. 20, 2012, pp. 6846-6860.

${ }^{10}$ Giles, M. B. and Süli, E., "Adjoint methods for PDEs: A posteriori error analysis and postprocessing by duality," Acta Numerica, Vol. 11, 2002, pp. 145-236.

${ }^{11}$ Fidkowski, K. J. and Darmofal, D. L., "Review of Output-Based Error Estimation and Mesh Adaptation in Computational Fluid Dynamics," AIAA Journal, Vol. 49, No. 4, 2011, pp. 673-694.

${ }^{12}$ Nordström, J., "The influence of open boundary conditions on the convergence to steady state for the Navier-Stokes equations," Journal of Computational Physics, Vol. 85, No. 1, 1989, pp. 210-244.

${ }^{13}$ Nordström, J., "The use of characteristic boundary conditions for the Navier-Stokes equations," Computers $E$ F Fluids, Vol. 24, No. 5, 1995, pp. 609-623.

${ }^{14}$ Nordström, J., Eriksson, S., and Eliasson, P., "Weak and Strong Wall Boundary Procedures and Convergence to SteadyState of the Navier-Stokes Equations," Journal of Computational Physics, Vol. 231, No. 14, 2012, pp. 4867-4884.

${ }^{15}$ Berg, J. and Nordström, J., "On the impact of boundary conditions on dual consistent finite difference discretizations," Journal of Computational Physics, Vol. 236, No. 0, 2013, pp. 41-55.

${ }^{16}$ Carpenter, M. H., Nordström, J., and Gottlieb, D., "A Stable and Conservative Interface Treatment of Arbitrary Spatial Accuracy," Journal of Computational Physics, Vol. 148, No. 2, 1999, pp. 341-365.

${ }^{17}$ Mattsson, K. and Nordström, J., "Summation by parts operators for finite difference approximations of second derivatives," Journal of Computational Physics, Vol. 199, No. 2, 2004, pp. 503-540.

${ }^{18}$ Mattsson, K., "Summation by Parts Operators for Finite Difference Approximations of Second-Derivatives with Variable Coefficients," Journal of Scientific Computing, Vol. 51, 2012, pp. 650-682.

${ }^{19}$ Strand, B., "Summation by Parts for Finite Difference Approximations for d/dx," Journal of Computational Physics, Vol. 110, No. 1, 1994, pp. 47-67.

${ }^{20}$ Svärd, M. and Nordström, J., "On the order of accuracy for difference approximations of initial-boundary value problems," Journal of Computational Physics, Vol. 218, No. 1, 2006, pp. 333-352.

${ }^{21}$ Hicken, J. E. and Zingg, D. W., "Summation-by-parts operators and high-order quadrature," Journal of Computational and Applied Mathematics, Vol. 237, No. 1, 2013, pp. 111-125.

${ }^{22}$ Svärd, M., Carpenter, M. H., and Nordström, J., "A stable high-order finite difference scheme for the compressible Navier-Stokes equations, far-field boundary conditions," Journal of Computational Physics, Vol. 225, No. 1, 2007, pp. 10201038.

${ }^{23}$ Kreiss, H.-O. and Lorenz, J., Initial-Boundary Value Problems and the Navier-Stokes Equations, SIAM, 2004.

${ }^{24}$ Abarbanel, S. and Gottlieb, D., "Optimal time splitting for two- and three-dimensional Navier-Stokes equations with mixed derivatives," Journal of Computational Physics, Vol. 41, No. 1, 1981, pp. 1-33.

${ }^{25}$ Nordström, J. and Svärd, M., "Well-Posed Boundary Conditions for the Navier-Stokes Equations," SIAM Journal on Numerical Analysis, Vol. 43, No. 3, 2005, pp. 1231-1255.

${ }^{26}$ Gustafsson, B., Kreiss, H.-O., and Oliger, J., Time Dependent Problems and Difference Methods, Wiley Interscience, 1995.

${ }^{27}$ Steinberg, S. and Roache, P. J., "Symbolic manipulation and computational fluid dynamics," Journal of Computational Physics, Vol. 57, No. 2, 1985, pp. 251-284.

${ }^{28}$ Shunn, L., Ham, F., and Moin, P., "Verification of variable-density flow solvers using manufactured solutions," Journal of Computational Physics, Vol. 231, No. 9, 2012, pp. 3801-3827.

${ }^{29}$ Berg, J. and Nordström, J., "Stable Robin solid wall boundary conditions for the Navier-Stokes equations," Journal of Computational Physics, Vol. 230, No. 19, 2011, pp. 7519-7532.

${ }^{30}$ Strikwerda, J. C., "Initial boundary value problems for incompletely parabolic systems," Communications on Pure and Applied Mathematics, Vol. 30, No. 6, 1977, pp. 797-822.

${ }^{31}$ Warming, R. F., Beam, R. M., and Hyett, B. J., "Diagonalization and Simultaneous Symmetrization of the Gas-Dynamic Matrices," Mathematics of Computation, Vol. 29, No. 132, 1975, pp. 1037-1045. 